\title{
Composição florística e fitossociologia de espécies arbóreas do Parque Fenológico da Embrapa Amazônia Ocidental
}

\author{
Kátia Emídio da SILVA ${ }^{1}$, Francisca Dionizia de Almeida MATOS², Marcelo Marques FERREIRA ${ }^{3}$
}

\begin{abstract}
RESUMO
Este trabalho objetiva avaliar a composição florística e a fitossociologia de espécies arbóreas do parque fenológico da Embrapa Amazônia Ocidental no Distrito Agropecuário da Suframa (DAS), Manaus-AM, a fim de subsidiar seleçôes futuras de árvores matrizes visando estudos fenológicos e a implantação de áreas de coleta de sementes. Foram alocadas aleatoriamente 20 parcelas de $10 \mathrm{~m}$ x $50 \mathrm{~m}$ ao longo de um transecto, amostrando-se todos os indivíduos com diâmetro a 1,30 m do solo, (DAP) $\geq 20,0$ $\mathrm{cm}$. Foram registrados 240 indivíduos, distribuídos em 100 espécies, 70 gêneros e 29 famílias. As famílias de maior importância ecológica são, em ordem decrescente, Lecythidaceae, Sapotaceae, Mimosaceae, Caesalpiniaceae, Chrysobalanaceae, Fabaceae, Humiriaceae, Moraceae, Vochysiaceae e Apocynaceae. Essas famílias contribuem com 67\% da riqueza local de espécies e com $75,8 \%$ do número de indivíduos, sugerindo que a diversidade vegetal da área está concentrada em poucas famílias. A família Lecythidaceae possui os maiores valores de dap e número de indivíduos, sendo Sapotaceae a que possui a maior riqueza de espécies na área. As espécies mais importantes, segundo o Índice de Valor de Importância-IVI, são Eschweilera coriacea (DC) S.A. Mori; Qualea paraensis Ducke; Vantanea macrocarpa Ducke; Eschweilera atropetiolata S.A. Mori; Couratari stellata A.C. Sm.; Lecythis usidata Miers; Eperua duckeana R.S. Cowan; Eschweilera amazonica R. Knuth e Chrysophyllum manaosense (Aubr.) T.D. Penn. O valor do índice de diversidade de Shannon de 2,71, aparentemente baixo, deve ser interpretado com cuidado ao se avaliar a diversidade do parque fenológico, uma vez que o diâmetro mínimo de $20,0 \mathrm{~cm}$ adotado neste trabalho pode ter restringido a identificação de um maior número de espécies. O índice de similaridade de Sorensen indicou uma alta dissimilaridade florística entre as parcelas. A primeira classe de diâmetro medindo de $20 \mathrm{~cm}-30 \mathrm{~cm}$ foi a que apresentou maior número de indivíduos, contendo $45 \%$ dos indivíduos amostrados. Acima do centro de classe de $55 \mathrm{~cm}$ de dap, cerca de 90\% das espécies estão representadas por apenas um indivíduo.
\end{abstract}

PalavraS-CHAVE: Fitossociologia, Diversidade de espécies vegetais, Parque fenológico, Terra Firme, Amazônia Central.

\section{Floristic composition and phytosociology of tree species in the Phenological Site of the Embrapa Western Amazonia}

\begin{abstract}
This research was carried out to study the floristic composition and phytosociology of tree species in the phenological site of Embrapa Western Amazonia, Suframa Agropecuary District-SAD, Manaus-AM, aiming to help future selection of seed trees, for the establishment of seed collecting areas. Twenty plots of $10 \mathrm{~m} \times 50 \mathrm{~m}$ were studied, along a topographic sequence, where trees with a diameter at breast height $(\mathrm{dbh}) \geq 20,0 \mathrm{~cm}$ were inventoried. A total of 240 trees belonging to 29 families, in 70 genera with 100 species were identified. The most important families, in a decreasing order, were: Lecythidaceae, Sapotaceae, Mimosaceae, Caesalpiniaceae, Chrysobalanaceae, Fabaceae, Humiriaceae, Moraceae, Vochysiaceae and Apocynaceae. These families constituted $67 \%$ of the local species richness and $75,8 \%$ of the number of individuals, suggesting that diversity is concentrated in a few families. Lecythidaceae had the largest $\mathrm{dbh}$ and number of individuals, and Sapotaceae the greatest richness species in the area. The most important species (IVIs) were Eschweilera coriacea (DC.) S.A. Mori; Qualea paraensis Ducke; Vantanea macrocarpa Ducke; Eschweilera atropetiolata S.A. Mori; Couratari stellata A.C. Sm.; Lecythis usidata Miers.; Eperua duckeana R.S. Cowan; Eschweilera amazonica R. Knuth and Chrysophyllum manaosense (Aubr.) T.D. Penn. The Shannon diversity and Sorensen similarity indexes indicated a low diversity and a high dissimilarity floristic among plots in this study for the minimum dbh considered. The diameter of the first class measuring $20 \mathrm{~cm}-30 \mathrm{~cm}$ presented the most number of individuals, contributing $45 \%$ of samples. Above the center class of $55 \mathrm{~cm}$ of dbh, around $90 \%$ of species have been represented by only one.
\end{abstract}

KEYWORDS: Phytosociology, Tree species diversity, Phenological site, Upland, Central Amazon.

1 Embrapa Amazônia Ocidental- AM 010, km 29, Cx. Postal 319, CEP 69048-660, Fone:(92) 3621-0300, Fax: (92) 3621-0430, Manaus-Amazonas e-mail: katia@cpaa.embrapa.br;

2 Instituto Nacional de Pesquisas da Amazônia- Manaus-AM- INPA/CPBO, Alameda Cosme Ferreira, 1756, Cx. Postal 478. CEP 69011-970. Fone: (92) 3643-3363,

Fax: (92) 3643-0303, Manaus, Amazonas. e-mail: fmatos@inpa.gov.br;

${ }^{3}$ Estudante de Eng. Florestal- Escola Superior de Tecnologia-EST, Av. Darcy Vargas, 1200, Parque 10 - CEP: 69065-020, Fone: (92) 3236-1470, Manaus-AM, e-mail: marceloutam@yahoo.com.br 


\section{INTRODUÇÃO}

O Brasil, com aproximadamente um terço das florestas tropicais remanescentes do mundo, é um dos mais importantes repositórios da biodiversidade mundial. Porém, o impacto das açōes antrópicas sobre os ambientes tem feito com que importantes ecossistemas sejam descaracterizados sem que se tenha conhecimento da sua estrutura fitossociológica e composição florística das espécies nos diferentes ambientes. $\mathrm{Na}$ Amazônia, muitas áreas são formadas por mosaicos de habitats, com diferentes conjuntos de espécies vegetais ocorrendo em áreas adjacentes sobre diferentes substratos, onde observase, de modo geral, que a distribuição das espécies arbóreas tropicais pode se dar devido a preferências de habitats e/ou a variaçôes na história evolutiva das mesmas (Pitman et al., 2001).

Estudos estruturais e florísticos desenvolvidos na Amazônia têm demonstrado que os ambientes florestais de terra firme apresentam alta diversidade, representada por poucos indivíduos de cada espécie (Prance et al., 1976; Lima Filho et al., 2001) e alta dissimilaridade florística entre parcelas adjacentes (Ferreira \& Prance, 1998). Assim, os crescentes desmatamentos de extensas áreas de cobertura vegetal na Amazônia, têm provocado perdas imensuráveis de recursos genéticos (Nobre et al., 1991; Houghton et al., 2000), onde espécies raras têm sido dizimadas e diversos habitats modificados pelas açôes antrópicas, afetando a resistência e resiliência dos ecossistemas. Considerando-se essa realidade, açôes que complementem ou gerem novos conhecimentos acerca da composição florística e estrutura fitossociológica de espécies de interesse comercial definido ou potencial, constituem-se em importantes medidas para minimizar a escassez de informações no setor florestal, contribuindo sobremaneira para a conservação e uso múltiplo sustentável da floresta.

Este trabalho objetiva contribuir para o maior conhecimento acerca da composição florística e estrutura fitossociológica de espécies arbóreas do parque fenológico da Embrapa Amazônia Ocidental, visando subsidiar açôes futuras de seleção de árvores matrizes para estudos fenológicos e implantação de áreas de coleta de sementes.

\section{MATERIAL E MÉTODOS}

$\mathrm{O}$ estudo foi desenvolvido em ambiente de terra firme, no parque fenológico do Campo Experimental da Embrapa Amazônia Ocidental, localizado na BR-174, Km 54, no Distrito Agropecuário da Suframa - DAS, Manaus-AM, o qual possui aproximadamente 400 ha.

O clima da área é do tipo "Am" na classificação de Köppen, com precipitação anual de 1.355 a $2.839 \mathrm{~mm}$. A temperatura média anual oscila entre 25,6 e $27,6^{\circ} \mathrm{C}$, com umidade relativa do ar variando de 84 a $90 \%$ ao longo do ano; os meses mais chuvosos vão de dezembro a maio, e os mais secos de agosto a novembro (RADAM, 1978).

Os solos predominantes na área são o latossolo amarelo com textura muito pesada e os hidromórficos, cobertos predominantemente pela vegetação da floresta densa de terras baixas, com dossel emergente (IBGE, 1999), consituídas por árvores que variam de médio a grande porte (Brazão et al., 1993). As terras firmes são planaltos formados por sedimentos da idade terciária que recobrem a maior extensão da Bacia Sedimentar Amazônica, apresentando topografias modeladas por formas de relevo dissecadas em amplos interflúvios tabulares e colinas (Regis, 1993).

$\mathrm{Na}$ área de estudo foram alocadas 20 parcelas de $10 \mathrm{~m} \mathrm{x}$ $50 \mathrm{~m}$ ao longo de um transecto de $10 \mathrm{~m} \times 1000 \mathrm{~m}$, lançado aleatoriamente na área, totalizando uma área amostral de $10.000 \mathrm{~m}^{2}$, contemplando as variaçōes da topografia da área (platô, encosta e baixio). Nessas parcelas foram amostrados todos os indivíduos com circunferência a 1,30m do solo (cap), igual ou maior que $63 \mathrm{~cm}$, correspondendo ao diâmetro a 1,30 $\mathrm{m}$ do solo, (dap) $\geq 20,0 \mathrm{~cm}$.

A identificação do material botânico foi realizada por meio de comparações com material depositado no herbário do Instituto Nacional de Pesquisas da Amazônia-INPA e por consulta à especialistas e à literatura especializada (Ribeiro et al., 1999), os quais adotam o sitema de classificação de Cronquist (1981). Os nomes botânicos apresentados estão de acordo com dados nomenclaturais do banco de dados do Missouri Botanical Garden (Mobot, 2005). Os indivíduos foram avaliados segundo sua distribuição diamétrica, utilizando-se o intervalo de classe de $10 \mathrm{~cm}$.

Os parâmetros fitossociológicos foram estimados segundo Müller-Dombois \& Ellemberg (1974), em conformidade com as fórmulas abaixo:

Densidade absoluta Densidade relativa

$$
D A_{i}=N_{i} \quad D R_{i}=\left(D A_{i} / \sum_{i=1}^{s} N_{i}\right) \times 100
$$

onde:

$\mathrm{N}_{\mathrm{i}}=$ número de indivíduos amostrados da i-ésima espécie;

$\sum_{i=1}^{s} N_{i}=$ número total de indivíduos amostrados;

Freqüência absoluta

Freqüência relativa

$F A_{i}=\left(N P_{i} / N P_{t}\right)^{*} 100$

onde:$$
F R_{i}=\left(F A_{i} / \sum_{i=1}^{s} F A_{i}\right) * 100
$$

$\mathrm{NP}_{\mathrm{i}}=$ número de parcelas em que ocorreu a i-ésima espécie;

$\mathrm{NP}_{\mathrm{t}}=$ número total de parcelas

$\sum_{i=1}^{t s} F A_{i}=$ somatório das frequências absolutas das espécies amostradas 
Dominância absoluta

$$
\operatorname{DoA}_{\mathrm{i}}=\mathrm{G}_{\mathrm{i}}
$$

Dominância relativa

onde:

$$
\operatorname{DoR}_{\mathrm{i}}=\left(\operatorname{DoA}_{\mathrm{i}=1} / \sum_{\mathrm{i}=1}^{S} \operatorname{DoA}_{\mathrm{i}}\right) * 100
$$

$\mathrm{G}_{\mathrm{i}}=$ área basal total da espécie $\mathrm{i}\left(\mathrm{m}^{2} \cdot \mathrm{ha}^{-1}\right)$

$\sum_{i=1}^{s} D \circ A_{i}=$ somatório das dominâncias absolutas de todas as espécies

Índice de valor de importância da espécies

$\mathrm{IVI}_{\mathrm{i}}=\sum_{i=1}^{s}\left(\mathrm{DR}_{\mathrm{i}}+\mathrm{FR}_{\mathrm{i}}+\mathrm{DoR}_{\mathrm{i}}\right)$

A importância ecológica das famílias no ecossistema foi estimada a partir do índice de valor de importância familiar (IVIF), sendo este o resultado da soma da diversidade ( $\mathrm{n}^{\circ}$ de espécies da família / $\mathrm{n}^{\circ}$ total de espécies), densidade e dominância relativas (Mori \& Boom, 1983).

A diversidade florística foi estimada de acordo com os índices de Riqueza de Espécie e de Diversidade de Shannon (Magurran, 1988). A Riqueza de Espécies foi obtida pela razão entre o número de espécies acumuladas e o tamanho da área amostrada $\left(\mathrm{m}^{2}\right)$, representada pela curva espécie-x-área. O índice de diversidade de Shannon foi obtido pela fórmula:

$\mathrm{H}^{\prime}=-\sum((\mathrm{pi})(\ln \mathrm{pi}))$, onde $\mathrm{pi}=(\mathrm{ni} / \mathrm{N})$ é a probabilidade de que um indivíduo amostrado pertença a espécie $\mathrm{i} ; \mathrm{ni}=\mathrm{n}^{\circ}$ total de indivíduos da espécie $\mathrm{i} ; \mathrm{N}=\mathrm{n}^{\circ}$ total de indivíduos amostrados na área.

Objetivando comparar a similaridade florística entre as 20 parcelas, foi construída uma matriz de presença e ausência das espécies e a partir dessa matriz foi calculada a similaridade florística através do índice qualitativo de Sorensen (MüllerDombois \& Ellemberg, 1974), por meio da fórmula :

$\mathrm{Cs}=(2 \mathrm{c} /(\mathrm{a}+\mathrm{b})) \times 100$, onde $\mathrm{c}=\mathrm{n}^{\circ}$ de espécies comuns às duas parcelas; $\mathrm{a} \mathrm{e} \mathrm{b}=\mathrm{n}^{\circ}$ de espécies presentes somente nas parcelas 1 e 2 , respectivamente.

Para interpretar a similaridade florística entre as parcelas utilizou-se o método de média de grupo (UPGMA) (Krebs, 1989), gerando-se um dendrograma no qual os valores das ordenadas expressam as relaçōes de similaridade entre os objetos (parcelas) indicadas nas abcissas. As análises foram feitas utilizando-se o software MVSP (Multi- variate Statistical Package).

\section{RESULTADOS E DISCUSSÃO}

\section{COMPOSIÇÃO FLORÍSTICA}

As 20 parcelas avaliadas estavam dispostas, segundo a posição topográfica, da seguinte forma: nove pertencem exclusivamente ao platô, três à encosta e somente uma ao baixio. Das parcelas restantes, três contemplaram a combinação de platô e encosta e quatro a combinação de encosta e baixio.
Foram registrados 240 indivíduos, distribuídos em 100 espécies, de 70 gêneros e 29 famílias. As espécies com maior número de indivíduos foram: Eschweilera coriacea (DC.) S.A. Mori, com 24 indivíduos; Eschweilera atropetiolata S.A. Mori com 9; Eperua duckeana Cowan, Qualea paraensis Ducke e Vantanea macrocarpa Ducke com 7 indivíduos cada; Eschweilera amazonica R. Knuth., Protium divaricatum Engl. e Sacoglotis matogrossensis Aubl., com 6, e Chrysophillum manaosense ( Aubr.) T.D. Penn. e Pouroma myrmecophila Ducke., com 5 indivíduos (Figura 1). Essas 10 espécies reúnem 34\% dos indivíduos registrados. Quarenta e nove porcento das espécies ocorrem na área com apenas um indivíduo, podendo-se citar Dinizia excelsa Ducke, Dipterix odorata (Aubl.) Willd., Parkia pendula (Willd.) Walp., Virola pavonis (A. DC.) A.C. Sm. e Goupia glabra Aubl., dentre outras.

(Number of individuals of the main species sampled at the phenological site of Embrapa in the Suframa Agropecuary District-SAD).

As famílias com maior número de espécies, em ordem decrescente, são: Sapotaceae (14), Lecythidaceae (9), Fabaceae (8), Caesalpiniaceae (7), Mimosaceae (7), Chrysobalanaceae (6) e Moraceae ( 6), (Figura 2). Essas sete famílias contribuíram com $57 \%$ da riqueza local de espécies e com aproximadamente $76 \%$ do número de indivíduos, sugerindo que a diversidade vegetal da área está concentrada em poucas famílias. Esse fato também foi observado por Matos \& Amaral (1999), Lima Filho et al. (2001) e EspíritoSanto et al. (2005). Oliveira \& Amaral (2005), estudando os aspectos florísticos, fitossociológicos e ecológicos de um sub-bosque de terra firme, na Estação Experimental do INPA$\mathrm{ZF}$ 2, área próxima à do presente estudo, também relataram o predominância das famílias Lecythidaceae, Sapotaceae e Fabaceae, dentre outras, em relação à diversidade e número de indivíduos. No dossel de florestas primárias, Nelson \& Oliveira (2001) e Pinto et al. (2003) também relataram a

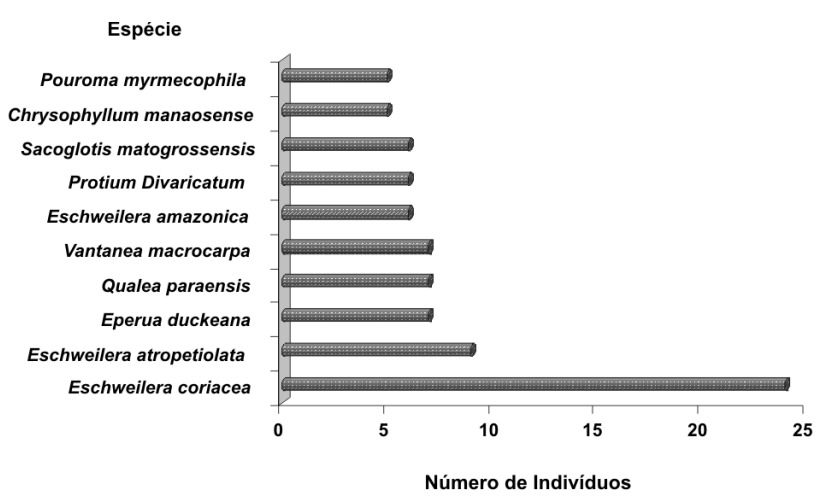

Figura 1 - Número de indivíduos das principais espécies amostradas no parque fenológico da Embrapa no Distrito Agropecuário da Suframa-DAS. 


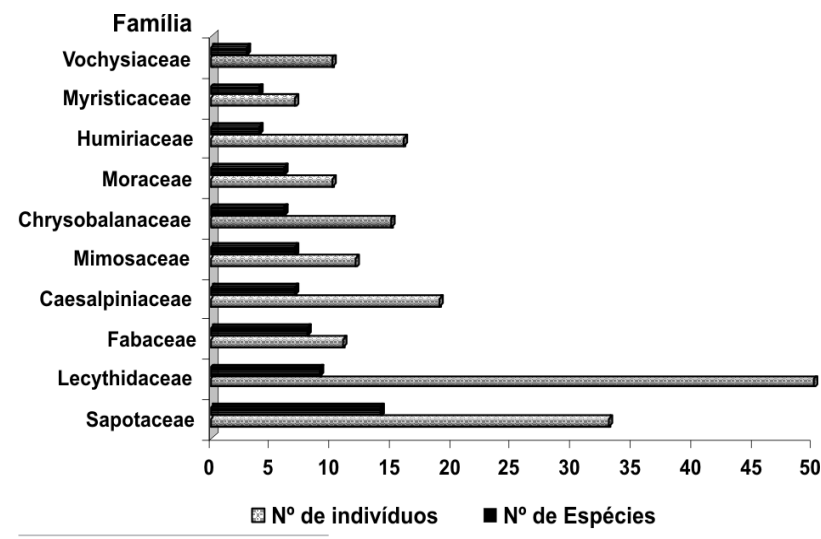

Figura 2 -Número de espécies e de indivíduos por família, amostrados no parque fenológico.

predominância, dentre outras, das famílias Lecythidaceae e Sapotaceae com relação à densidade de indivíduos.

\section{FITOSSOCIOLOGIA}

O Índice de Valor de Importância (IVI) calculado para as espécies é um indicador da importância ecológica das mesmas, devido a influência relativa das espécies mais frequentes e dominantes nos processos de equilíbrio da flora e manutenção da fauna, fornecendo abrigo e alimentação (Oliveira \& Amaral, 2003).

As espécies com os 10 maiores valores de IVI, em ordem decrescente, foram: Eschweilera coriacea, Qualea paraensis, Vantanea macrocarpa, Eschweilera atropetiolata, Couratari stellata, Lecythis usidata, Eschweilera amazonica, Eperua duckeana, Chrysophyllum manaosense e Protium divaricatum (Figura 3 e Tabela 1). Nestas espécies estão concentrados $29,0 \%$ dos indivíduos amostrados e $88 \%$ dos valores de IVI, evidenciando a importância das mesmas na área em estudo. Tais espécies são citadas com freqüência em diversos trabalhos desenvolvidos em ecossistemas de terra firme da Amazônia (Amaral, 1996; Lima Filho et al., 2001). Eschweilera coriacea e Vantanea macrocarpa são as espécies com melhor distribuição horizontal na área, com 65\% e 35\% para os valores de freqüência, respectivamente.

As famílias com os maiores valores para o índice de importância familiar (IVIF), em ordem decrescente, foram: Lecythidaceae, Sapotaceae, Mimosaceae, Caesalpiniaceae, Chrysobalanaceae, Fabaceae, Humiriaceae, Moraceae, Vochysiaceae e Apocynaceae (Figura 4). A família Lecythidaceae apresentou os maiores valores de dap, bem como de número de indivíduos, enquanto a família Sapotaceae apresentou a maior diversidade de espécies. Em estudo realizado por Oliveira \& Amaral (2003), na Estação Experimental ZF-2/INPA, em área florestal de terra firme, os autores registraram também os maiores valores de IVIF para Lecythidaceae e Sapotaceae, nesta ordem, bem como para a maioria das famílias aqui destacadas.

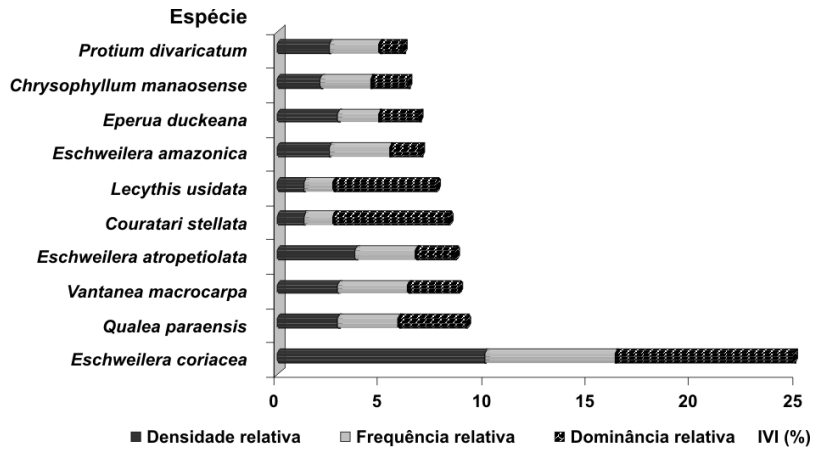

Figura 3 - Composição do Índice de Valor de Importância - IVI para as espécies com os maiores valores de IVI no parque fenológico.

\section{DIVERSIDADE E SIMILARIDADE FLORÍSTICA}

A diversidade calculada pelo índice de Shannon, segundo alguns estudos realizados em florestas tropicais, varia de 3,83 a 5,85 (Knight, 1975). Para o critério adotado neste trabalho, o índice obtido foi da ordem de 2,71, sugerindo baixa diversidade local, considerando-se o valor mínimo de dap de $20,0 \mathrm{~cm}$. Ao se analisar a curva espécie-x-área (Figura 5) observa-se uma tendência de estabilização da mesma, uma vez que o ingresso de espécies novas por meio do acréscimo da área amostral reduziu-se bastante a partir de 0,8 ha. Entretanto, esta estabilização não se concretizou, sugerindo que o esforço amostral de 1,0 hectare para se avaliar a diversidade do parque fenológico, considerando-se indivíduos com o dap $\geq 20,0 \mathrm{~cm}$, ainda não foi suficiente, necessitando de estudos adicionais com número maior de parcelas. Deve-se destacar, entretanto, que para se avaliar a diversidade e similaridade florística do parque fenológico, como um todo, o nível inclusão de dap deve ser inferior ao adotado neste trabalho.

A Figura 6 mostra o dendrograma obtido para as parcelas, utilizando-se o método média de grupo (UPGMA), com base no índice de Sorensen. Observa-se de modo geral a não formação de grupos entre as parcelas, indicando uma dissimilaridade florística entre elas. A maior similaridade

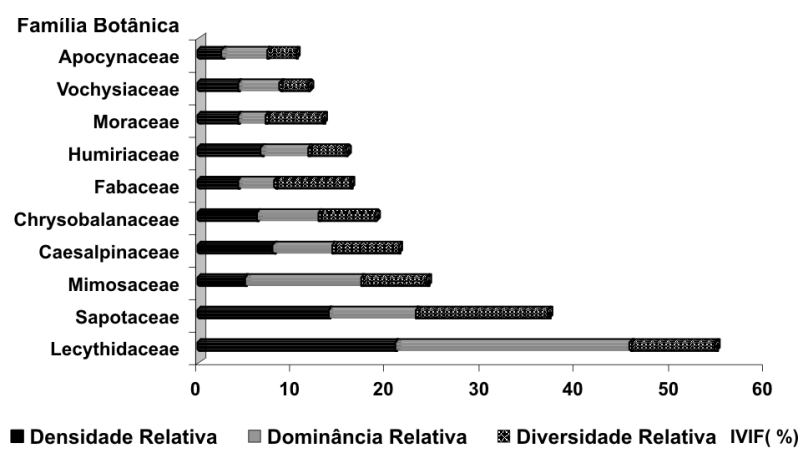

Figura 4 - Composição do Índice de Valor de Importância Familiar - IVIF para as famílias com maiores valores, no parque fenológico-DAS. 
Tabela 1 - Relação das espécies arbóreas inventariadas e seus respectivos atributos: DA = número de indivíduos da espécie $i ;$ FA = número de parcelas ocorrentes da espécie $i ; \mathrm{DoA}=$ dominância absoluta; $\mathrm{DR}=$ densidade relativa; $\mathrm{FR}=$ freqüência relativa; $\mathrm{DoR}=$ dominância relativa; $\mathrm{IVI}=$ índice de valor de importância (DR+FR+DoR).

\begin{tabular}{|c|c|c|c|c|c|c|c|}
\hline Família / Espécie & DA & FA & DoA & $\begin{array}{l}\text { DR } \\
\text { (\%) }\end{array}$ & $\begin{array}{l}\text { FR } \\
(\%)\end{array}$ & $\begin{array}{l}\text { DoR } \\
\text { (\%) }\end{array}$ & $\begin{array}{l}\text { IVI } \\
\text { (\%) }\end{array}$ \\
\hline \multicolumn{8}{|l|}{ Anisophylleaceae } \\
\hline Anisophyllea manausensis Pires \& W.A Rodr. & 1 & 1 & 0,13 & 0,42 & 0,48 & 0,40 & 1,30 \\
\hline \multicolumn{8}{|l|}{ Annonaceae } \\
\hline Unonopsis guatterioides (A.DC.) R.E. Fr. & 2 & 1 & 0,09 & 0,83 & 0,48 & 0,27 & 1,59 \\
\hline Unonopsis duckei R.E. Fr. & 1 & 1 & 0,08 & 0,42 & 0,48 & 0,26 & 1,16 \\
\hline Bocageopsis multiflora (Mart.) R.E. Fr. & 1 & 1 & 0,05 & 0,42 & 0,48 & 0,16 & 1,05 \\
\hline \multicolumn{8}{|l|}{ Apocynaceae } \\
\hline Aspidosperma marcgravianum Woodson & 2 & 2 & 0,89 & 0,83 & 0,97 & 2,79 & 4,59 \\
\hline Couma utilis (Mart.) Müll. Arg. & 2 & 2 & 0,37 & 0,83 & 0,97 & 1,16 & 2,96 \\
\hline Geissospermum argenteum Woodson & 2 & 1 & 0,28 & 0,83 & 0,48 & 0,87 & 2,19 \\
\hline \multicolumn{8}{|l|}{ Bignoniaceae } \\
\hline Tabebuia serratifolia (Vahl) G. Nicholson & 1 & 1 & 0,06 & 0,42 & 0,48 & 0,19 & 1,09 \\
\hline \multicolumn{8}{|l|}{ Bombacaceae } \\
\hline Scleronema micranthum (Ducke) Ducke & 3 & 3 & 0,52 & 1,25 & 1,45 & 1,62 & 4,32 \\
\hline \multicolumn{8}{|l|}{ Burseraceae } \\
\hline Protium divaricatum Engl. & 6 & 5 & 0,37 & 2,50 & 2,42 & 1,14 & 6,06 \\
\hline Trattinnickia rhoifolia Willd. & 1 & 1 & 0,05 & 0,42 & 0,48 & 0,14 & 1,04 \\
\hline \multicolumn{8}{|l|}{ Caesalpinaceae } \\
\hline Eperua duckeana R.S. Cowan & 7 & 4 & 0,63 & 2,92 & 1,93 & 1,97 & 6,82 \\
\hline Eperua glabiflora (Ducke) R.S. Cowan & 4 & 4 & 0,55 & 1,67 & 1,93 & 1,71 & 5,31 \\
\hline Macrolobium suavelolens Spruce ex Benth. & 4 & 3 & 0,31 & 1,67 & 1,45 & 0,97 & 4,09 \\
\hline Dialium guianense Steud. & 1 & 1 & 0,20 & 0,42 & 0,48 & 0,61 & 1,51 \\
\hline Sclerolobium melanocarpum Ducke & 1 & 1 & 0,13 & 0,42 & 0,48 & 0,39 & 1,29 \\
\hline Eperua bijuga Mart. ex Benth. & 1 & 1 & 0,10 & 0,42 & 0,48 & 0,30 & 1,20 \\
\hline Peltogyne paniculata Benth. & 1 & 1 & 0,08 & 0,42 & 0,48 & 0,25 & 1,15 \\
\hline \multicolumn{8}{|l|}{ Cecropiaceae } \\
\hline Pouroma myrmecophyla Ducke & 5 & 4 & 0,36 & 2,08 & 1,93 & 1,13 & 5,15 \\
\hline Pourouma minor Benoist & 1 & 1 & 0,08 & 0,42 & 0,48 & 0,25 & 1,15 \\
\hline \multicolumn{8}{|l|}{ Celastraceae } \\
\hline Goupia glabra Aubl. & 1 & 1 & 0,06 & 0,42 & 0,48 & 0,19 & 1,09 \\
\hline \multicolumn{8}{|l|}{ Chrysobalanaceae } \\
\hline Licania laevigata Prance & 4 & 4 & 0,75 & 1,67 & 1,93 & 2,33 & 5,93 \\
\hline Licania octandra (Hoffmanns ex R. \& S.) Kuntze & 4 & 4 & 0,45 & 1,67 & 1,93 & 1,42 & 5,02 \\
\hline Couepia Iongipendula Pilg. & 3 & 3 & 0,25 & 1,25 & 1,45 & 0,77 & 3,47 \\
\hline Licania heteromorpha Benth. & 2 & 2 & 0,52 & 0,83 & 0,97 & 1,62 & 3,42 \\
\hline Licania micrantha Miq. & 1 & 1 & 0,05 & 0,42 & 0,48 & 0,16 & 1,06 \\
\hline Licania oblongifolia Standl. & 1 & 1 & 0,04 & 0,42 & 0,48 & 0,14 & 1,04 \\
\hline \multicolumn{8}{|l|}{ Combretaceae } \\
\hline Buchenavia grandis Ducke & 1 & 1 & 1,47 & 0,42 & 0,48 & 4,59 & 5,49 \\
\hline Buchenavia parvifolia Ducke & 1 & 1 & 0,09 & 0,42 & 0,48 & 0,28 & 1,18 \\
\hline \multicolumn{8}{|l|}{ Euphorbiaceae } \\
\hline Conceveiba guianensis Aubl. & 3 & 2 & 0,14 & 1,25 & 0,97 & 0,45 & 2,66 \\
\hline Pogonophora schomburgkiana Miers ex Benth. & 2 & 2 & 0,12 & 0,83 & 0,97 & 0,38 & 2,18 \\
\hline
\end{tabular}




\begin{tabular}{|c|c|c|c|c|c|c|c|}
\hline Família / Espécie & DA & FA & DoA & $\begin{array}{l}\text { DR } \\
\text { (\%) }\end{array}$ & $\begin{array}{l}\text { FR } \\
(\%)\end{array}$ & $\begin{array}{c}\text { DoR } \\
\text { (\%) }\end{array}$ & $\begin{array}{l}\text { IVI } \\
\text { (\%) }\end{array}$ \\
\hline Swartzia ingifolia Ducke & 2 & 2 & 0,13 & 0,83 & 0,97 & 0,40 & 2,20 \\
\hline Ormosia coccinea (Aubl.) Jacks. & 1 & 1 & 0,41 & 0,42 & 0,48 & 1,29 & 2,19 \\
\hline Andira trifoliolata Ducke & 2 & 1 & 0,09 & 0,83 & 0,48 & 0,28 & 1,60 \\
\hline Taralea oppositifolia Aubl. & 1 & 1 & 0,22 & 0,42 & 0,48 & 0,68 & 1,58 \\
\hline Hymenelobium sericeum Ducke & 1 & 1 & 0,13 & 0,42 & 0,48 & 0,41 & 1,31 \\
\hline Ormosia grossa Rudd & 1 & 1 & 0,09 & 0,42 & 0,48 & 0,30 & 1,19 \\
\hline Dipteryx odorata (Aubl.) Willd. & 1 & 1 & 0,08 & 0,42 & 0,48 & 0,25 & 1,15 \\
\hline Vatairea sericea Ducke & 1 & 1 & 0,05 & 0,42 & 0,48 & 0,17 & 1,07 \\
\hline Paramachaerium ormosioides (Ducke) Ducke & 1 & 1 & 0,04 & 0,42 & 0,48 & 0,14 & 1,04 \\
\hline \multicolumn{8}{|l|}{ Flacourtiaceae } \\
\hline Laetia procera (Poepp.) Eichler & 1 & 1 & 0,14 & 0,42 & 0,48 & 0,43 & 1,33 \\
\hline \multicolumn{8}{|l|}{ Humiriaceae } \\
\hline Endopleura uchi (Huber) Cuatrec. & 2 & 2 & 0,17 & 0,83 & 0,97 & 0,52 & 2,32 \\
\hline Sacoglottis guianensis Benth. & 1 & 1 & 0,04 & 0,42 & 0,48 & 0,14 & 1,04 \\
\hline Sacoglottis mattogrossensis Malme & 6 & 3 & 0,62 & 2,50 & 1,45 & 1,94 & 5,89 \\
\hline Vantanea macrocarpa Ducke & 7 & 7 & 0,76 & 2,92 & 3,38 & 2,38 & 8,68 \\
\hline \multicolumn{8}{|l|}{ Lauraceae } \\
\hline Licaria macrophylla (A.C.SM.) Kost. & 1 & 1 & 0,07 & 0,42 & 0,48 & 0,22 & 1,12 \\
\hline Ocotea guianensis Aubl. & 2 & 2 & 0,11 & 0,83 & 0,97 & 0,35 & 2,15 \\
\hline \multicolumn{8}{|l|}{ Lecythidaceae } \\
\hline Eschweilera coriacea (DC.) S.A. Mori & 24 & 13 & 2,75 & 10,0 & 6,28 & 8,58 & 24,86 \\
\hline Eschweilera atropetiolata S.A. Mori & 9 & 6 & 0,60 & 3,75 & 2,90 & 1,88 & 8,53 \\
\hline Couratari stellata A.C.Sm. & 3 & 3 & 1,78 & 1,25 & 1,45 & 5,55 & 8,24 \\
\hline Lecythis usidata Miers & 3 & 3 & 1,57 & 1,25 & 1,45 & 4,91 & 7,61 \\
\hline Eschweilera amazonica R. Knuth & 6 & 6 & 0,48 & 2,50 & 2,90 & 1,51 & 6,90 \\
\hline Couratari guianensis Aubl. & 2 & 2 & 0,59 & 0,83 & 0,97 & 1,84 & 3,64 \\
\hline Lecythis prance S.A. Mori & 2 & 2 & 0,12 & 0,83 & 0,97 & 0,36 & 2,16 \\
\hline Lecythis lurida (Miers.)Mori & 1 & 1 & 0,06 & 0,42 & 0,48 & 0,20 & 1,10 \\
\hline \multicolumn{8}{|l|}{ Melastomataceae } \\
\hline Miconia regelii Cogn. & 1 & 1 & 0,07 & 0,42 & 0,48 & 0,22 & 1,12 \\
\hline \multicolumn{8}{|l|}{ Memecylaceae } \\
\hline Mouriri angulicosta Morley & 2 & 2 & 0,24 & 0,83 & 0,97 & 0,76 & 2,56 \\
\hline Mouriri huberi Cogn. & 1 & 1 & 0,04 & 0,42 & 0,48 & 0,14 & 1,04 \\
\hline \multicolumn{8}{|l|}{ Mimosaceae } \\
\hline Dinizia excelsa Ducke & 1 & 1 & 1,40 & 0,42 & 0,48 & 4,38 & 5,28 \\
\hline Piptadenia suaveolens Miq. & 1 & 1 & 1,27 & 0,42 & 0,48 & 3,98 & 4,88 \\
\hline Inga alba (Sw.) Willd. & 3 & 2 & 0,20 & 1,25 & 0,97 & 0,64 & 2,85 \\
\hline Parkia pendula (Willd.) Walp. & 1 & 1 & 0,62 & 0,42 & 0,48 & 1,95 & 2,85 \\
\hline Parkia multijuga Benth. & 3 & 2 & 0,17 & 1,25 & 0,97 & 0,52 & 2,74 \\
\hline Inga heterophylla Willd. & 2 & 2 & 0,18 & 0,83 & 0,97 & 0,57 & 2,37 \\
\hline Stryphnodendron guianensis (Aubl.) Benth. & 1 & 1 & 0,04 & 0,42 & 0,48 & 0,14 & 1,04 \\
\hline \multicolumn{8}{|l|}{ Moraceae } \\
\hline Helicostylis scabra (J.F. Macbr.) C.C. Berg & 3 & 3 & 0,29 & 1,25 & 1,45 & 0,92 & 3,62 \\
\hline Brosimum utile (Kunth) Oken ex J. Presl & 2 & 2 & 0,43 & 0,83 & 0,97 & 1,33 & 3,13 \\
\hline Clarisia racemosa Ruiz e Pav. & 1 & 1 & 0,08 & 0,42 & 0,48 & 0,26 & 1,16 \\
\hline Brosimum rubescens Taub. & 1 & 1 & 0,07 & 0,42 & 0,48 & 0,22 & 1,12 \\
\hline Naucleopsis caloneura (Huber) Ducke & 1 & 1 & 0,05 & 0,42 & 0,48 & 0,16 & 1,06 \\
\hline
\end{tabular}




\begin{tabular}{|c|c|c|c|c|c|c|c|}
\hline Família / Espécie & DA & FA & DoA & $\begin{array}{l}\text { DR } \\
\text { (\%) }\end{array}$ & $\begin{array}{l}\text { FR } \\
\text { (\%) }\end{array}$ & $\begin{array}{l}\text { DoR } \\
\text { (\%) }\end{array}$ & $\begin{array}{l}\text { IVI } \\
\text { (\%) }\end{array}$ \\
\hline Naucleopsis imitans (Ducke) C.C. Berg & 1 & 1 & 0,04 & 0,42 & 0,48 & 0,12 & 1,02 \\
\hline \multicolumn{8}{|l|}{ Myristicaceae } \\
\hline Osteophloeum plastyspermum (A. DC.) Warb. & 3 & 3 & 0,86 & 1,25 & 1,45 & 2,68 & 5,37 \\
\hline Virola calophylla (Spruce) Warb. & 2 & 2 & 0,12 & 0,83 & 0,97 & 0,37 & 2,17 \\
\hline Iryanthera juruensis Warb. & 1 & 1 & 0,06 & 0,42 & 0,48 & 0,19 & 1,09 \\
\hline Virola pavonis (A. DC.) A.C. Sm. & 1 & 1 & 0,05 & 0,42 & 0,48 & 0,16 & 1,06 \\
\hline \multicolumn{8}{|l|}{ Olacaceae } \\
\hline Minquartia guianensis Aubl. & 4 & 3 & 0,25 & 1,67 & 1,45 & 0,77 & 3,89 \\
\hline Heisteria duckei Engl. & 1 & 1 & 0,06 & 0,42 & 0,48 & 0,19 & 1,09 \\
\hline \multicolumn{8}{|l|}{ Rutaceae } \\
\hline Hortia Iongifolia Ducke & 1 & 1 & 0,10 & 0,42 & 0,48 & 0,31 & 1,21 \\
\hline \multicolumn{8}{|l|}{ Sapindaceae } \\
\hline Toulicia pulvinata Radlk. & 1 & 1 & 0,07 & 0,42 & 0,48 & 0,22 & 1,12 \\
\hline Talisia cupularis Radlk. & 1 & 1 & 0,05 & 0,42 & 0,48 & 0,15 & 1,05 \\
\hline \multicolumn{8}{|l|}{ Sapotaceae } \\
\hline Chrysophyllum manaosense ( Aubr.) T.D. Penn. & 5 & 5 & 0,57 & 2,08 & 2,42 & 1,79 & 6,29 \\
\hline Pouteria guianensis Aubl. & 4 & 4 & 0,49 & 1,67 & 1,93 & 1,53 & 5,13 \\
\hline Pouteria oblanceolata Pires & 4 & 4 & 0,28 & 1,67 & 1,93 & 0,87 & 4,47 \\
\hline Chrysophyllum prieurii A. DC. & 4 & 3 & 0,30 & 1,67 & 1,45 & 0,95 & 4,06 \\
\hline Pouteria minima T.D. Penn. & 3 & 3 & 0,21 & 1,25 & 1,45 & 0,64 & 3,34 \\
\hline Micropholis cylindrocapa (Poepp.) Pierre & 3 & 2 & 0,19 & 1,25 & 0,97 & 0,58 & 2,80 \\
\hline Manilkara bidentata (A. DC) A. Chev. & 2 & 2 & 0,23 & 0,83 & 0,97 & 0,73 & 2,53 \\
\hline Chrysophyllum sanguinolentum (Pierre) Baehni & 2 & 2 & 0,22 & 0,83 & 0,97 & 0,69 & 2,49 \\
\hline Micropholis guyanensis (A. DC.) Pierre & 2 & 2 & 0,19 & 0,83 & 0,97 & 0,60 & 2,40 \\
\hline Pouteria caimito (Ruiz \& Pav.) Radlk. & 1 & 1 & 0,09 & 0,42 & 0,48 & 0,28 & 1,18 \\
\hline Pouteria venosa (Mart.) Baehni & 1 & 1 & 0,09 & 0,42 & 0,48 & 0,28 & 1,18 \\
\hline Micropholis venulosa (Mart. \& Eichler) Pierre & 1 & 1 & 0,05 & 0,42 & 0,48 & 0,15 & 1,05 \\
\hline Pouteria petiolata T.D. Penn & 1 & 1 & 0,04 & 0,42 & 0,48 & 0,13 & 1,03 \\
\hline \multicolumn{8}{|l|}{ Tiliaceae } \\
\hline Luheopsis rosea (Ducke) Burret & 2 & 2 & 0,21 & 0,83 & 0,97 & 0,67 & 2,47 \\
\hline Apeiba echinata Gaertner & 2 & 2 & 0,13 & 0,83 & 0,97 & 0,41 & 2,21 \\
\hline \multicolumn{8}{|l|}{ Violaceae } \\
\hline Rinorea guianensis Aubl. & 2 & 2 & 0,09 & 0,83 & 0,97 & 0,28 & 2,08 \\
\hline \multicolumn{8}{|l|}{ Vochysiaceae } \\
\hline Qualea paraensis Ducke & 7 & 6 & 1,04 & 2,92 & 2,90 & 3,25 & 9,07 \\
\hline Vochysia inundata Ducke & 2 & 2 & 0,25 & 0,83 & 0,97 & 0,79 & 2,59 \\
\hline Vochysia guianensis Aubl. & 1 & 1 & 0,13 & 0,42 & 0,48 & 0,42 & 1,32 \\
\hline
\end{tabular}

foi observada entre as parcelas 11 e 15, com um índice de 37,5 , valor este que pode ser considerado baixo, quando comparados aos resultados obtidos por Oliveira \& Mori (1999) e Oliveira \& Amaral (2003). O número de espécies por parcela varia de 6 a 15 espécies $/ 500 \mathrm{~m}^{2}$, com desvio padrão de 2,76, mostrando homogeneidade no número de espécies, aliado a uma baixa similaridade entre as parcelas segundo o índice de Sorensen. O diâmetro mínimo estabelecido para o presente trabalho pode ter desempenhado papel importante na baixa densidade de indivíduos e baixo número de espécies observadas na maioria das parcelas, o que pode ter afetado os índices calculados, uma vez que $49 \%$ das espécies foram registradas com apenas um indivíduo.

\section{DISTRIBUICCÃO DIAMÉTRICA}

A distribuição dos indivíduos nas classes diamétricas fornece importantes informaçōes sobre a estrutura da floresta, que aliada a atributos qualitativos dos indivíduos 


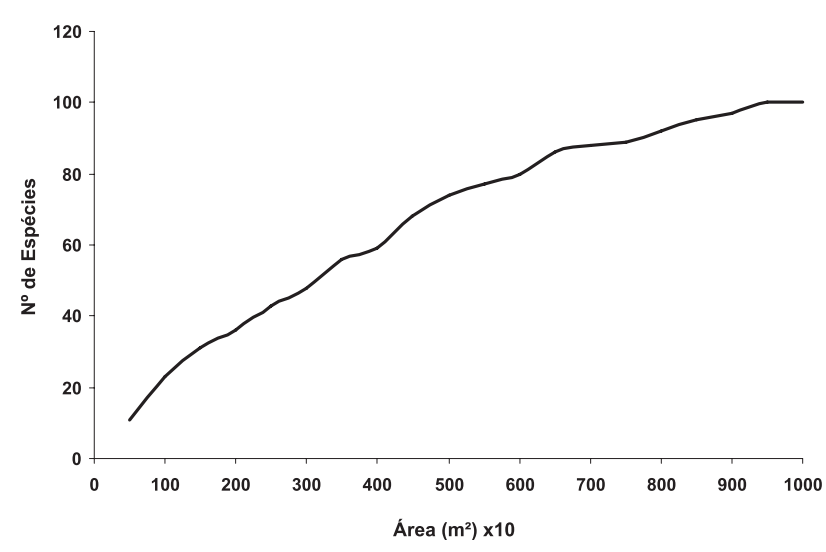

Figura 5 - Curva cumulativa de espécies com DAP $>20,0 \mathrm{~cm}$, versus área amostrada no parque fenológico. (Cumulative curve of species with DBH $\geq$ $20,0 \mathrm{~cm}$, versus sampled area at the phenological site).

são importantes subsídios ao processo de seleção de árvores matrizes.

Observa-se na Figura 7, que aproximadamente 45\% dos indivíduos estão distribuídos na classe de $20-30 \mathrm{~cm}$. As três primeiras classes $(20 \mathrm{~cm}$ a $50 \mathrm{~cm})$ concentraram aproximadamente $88 \%$ do número total de indivíduos amostrados.

Ao analisar a Tabela 2, com os valores de área basal e número de indivíduos distribuídos em 9 classes diamétricas

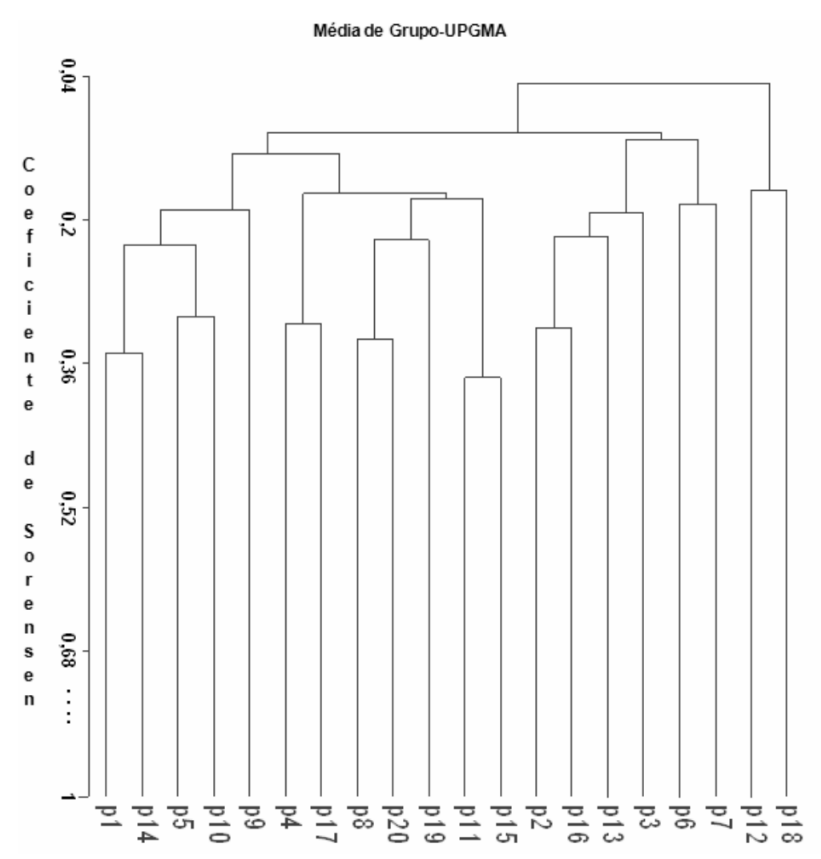

Figura 6 - Dendrograma obtido a partir do Índice de Similaridade de Sorensen, para os dados de presença e ausência das espécies, nas 20 parcelas de floresta densa do parque fenológico. para as 20 espécies com os maiores valores de IVI, observa-se que estas espécies reproduziram o mesmo padrão observado para toda a área amostrada, com maior concentração de indivíduos na classe $20-30 \mathrm{~cm}$ e as três primeiras classes reunindo aproximadamente $84 \%$ dos indivíduos pertencentes às 20 espécies. E. coriacea apresenta o maior valor de área basal, com indivíduos distribuídos em quatro classes diamétricas, com maior concentração destes na classe de 20-30 cm. A partir do centro de classe de $55 \mathrm{~cm}$, as espécies aparecem com apenas um indivíduo. Não foi observada a presença de indivíduos nos centros de classe $85 \mathrm{~cm}$ e $95 \mathrm{~cm}$.

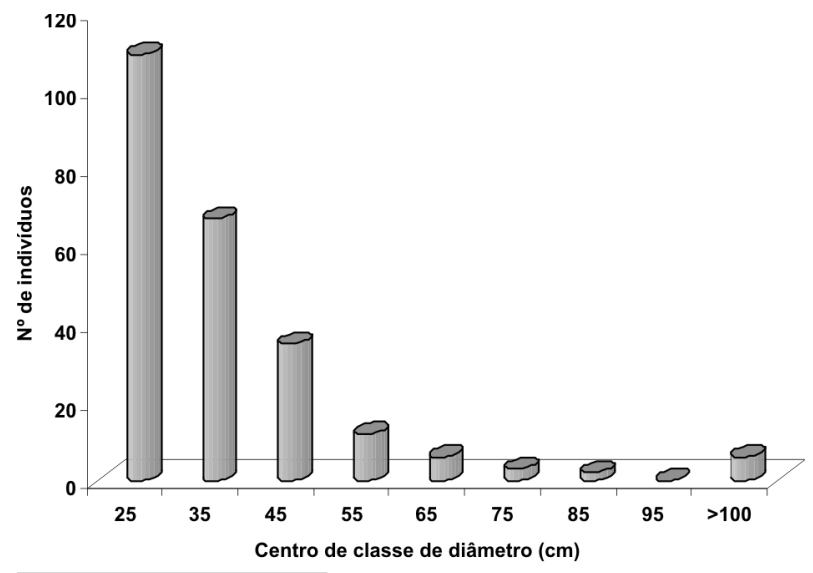

Figura 7- Número total de indivíduos, por classe diamétrica, para as espécies amostradas no parque fenológico.

\section{CONCLUSÕES}

O parque fenológico da Embrapa Amazônia Ocidental - DAS, avaliado segundo a florística e fitossociologia das espécies, apresenta baixa densidade de indivíduos por espécie, considerando-se o dap mínimo de $20 \mathrm{~cm}$. Essa baixa densidade, observada em $49 \%$ das espécies, contribuiu para a baixa diversidade e a alta dissimilaridade entre as parcelas amostrais.

No processo de seleção de árvores matrizes para estudos fenológicos e coleta de sementes, as espécies com os maiores valores de IVI devem ser avaliadas prioritariamente, uma vez que congregam atributos importantes, tais como densidade, freqüência e dominância, podendo-se citar as espécies Eschweilera coriacea, Qualea paraensis, Vantanea macrocarpa, dentre outras. Espécies com baixa densidade/ha (1 indivíduo), conforme observado neste trabalho, podem representar a necessidade do estabelecimento de extensas áreas de estudo, com maiores custos financeiros e operacionais, a fim de se ter uma maior representatividade dos resultados obtidos. $\mathrm{O}$ conhecimento da composição e estrutura de comunidades 
Tabela 2 - Distribuição da área basal- G (m2 /ha) e do número de indivíduos (N) em classes diamétricas, de amplitude de 10cm, para as espécies com os maiores valores de IVI.

\begin{tabular}{|c|c|c|c|c|c|c|c|c|c|c|c|}
\hline \multirow{2}{*}{ Espécies } & & \multicolumn{8}{|c|}{ Classes de DAP (cm) } & \multirow[b]{2}{*}{$>100$} & \multirow[b]{2}{*}{ Total } \\
\hline & & $20-30$ & $30-40$ & $40-50$ & $50-60$ & $60-70$ & $70-80$ & $80-90$ & $90-100$ & & \\
\hline \multirow[t]{2}{*}{ Eschweilera coriacea (DC.) S.A. Mori } & $\mathrm{G}$ & 0,7857 & 0,6247 & & 0,4714 & & & & & 0,8666 & 2,7485 \\
\hline & $\mathrm{N}$ & 15 & 6 & & 2 & & & & & 1 & 24 \\
\hline \multirow[t]{2}{*}{ Qualea paraensis Ducke } & $\mathrm{G}$ & 0,0561 & 0,2460 & 0,1472 & 0,2578 & 0,3344 & & & & & 1,0416 \\
\hline & $\mathrm{N}$ & 1 & 3 & 1 & 1 & 1 & & & & & 7 \\
\hline \multirow[t]{2}{*}{ Vantanea macrocarpa Ducke } & $\mathrm{G}$ & 0,1348 & 0,1681 & 0,4591 & & & & & & & 0,7620 \\
\hline & N & 2 & 2 & 3 & & & & & & & 7 \\
\hline \multirow[t]{2}{*}{ Eschweilera atropetiolata S.A. Mori } & G & 0,3064 & 0,2952 & & & & & & & & 0,6016 \\
\hline & N & 6 & 3 & & & & & & & & 9 \\
\hline \multirow[t]{2}{*}{ Couratari stellata A.C.Sm. } & G & & & 0,1767 & 0,2088 & & & & & 1,3904 & 1,7759 \\
\hline & N & & & 1 & 1 & & & & & 1 & 3 \\
\hline \multirow[t]{2}{*}{ Lecythis usidata Miers } & G & & & 0,2927 & & & & & & 1,2796 & 1,5723 \\
\hline & N & & & 2 & & & & & & 1 & 3 \\
\hline \multirow[t]{2}{*}{ Eschweilera amazonica R. Knuth } & G & 0,0956 & 0,3866 & & & & & & & & 0,4822 \\
\hline & N & 2 & 4 & & & & & & & & 6 \\
\hline \multirow[t]{2}{*}{ Eperua duckeana R.S. Cowan } & G & 0,1865 & 0,1824 & 0,2608 & & & & & & & 0,6296 \\
\hline & N & 3 & 2 & 2 & & & & & & & 7 \\
\hline \multirow[t]{2}{*}{ Chrysophyllum manaosense (Aubr.) T.D. Penn } & $\mathrm{G}$ & 0,0497 & 0,2733 & & 0,2493 & & & & & & 0,5723 \\
\hline & $\mathrm{N}$ & 1 & 3 & & 1 & & & & & & 5 \\
\hline \multirow[t]{2}{*}{ Protium divaricatum Engl. } & G & 0,2698 & 0,0963 & & & & & & & & 0,3661 \\
\hline & $\mathrm{N}$ & 5 & 1 & & & & & & & & 6 \\
\hline \multirow[t]{2}{*}{ Licania laevigata Prance } & G & 0,0522 & & 0,1408 & 0,2665 & 0,2873 & & & & & 0,7467 \\
\hline & $\mathrm{N}$ & 1 & & 1 & 1 & 1 & & & & & 4 \\
\hline \multirow[t]{2}{*}{ Sacoglottis mattogrossensis Malme } & G & 0,1251 & 0,0796 & 0,1863 & 0,2300 & & & & & & 0,6210 \\
\hline & N & 3 & 1 & 1 & 1 & & & & & & 6 \\
\hline \multirow[t]{2}{*}{ Buchenavia grandis Ducke } & $\mathrm{G}$ & & & & & & & & & 1,4714 & 1,4714 \\
\hline & N & & & & & & & & & 1 & 1 \\
\hline \multirow[t]{2}{*}{ Osteophloeum plastyspermum (A. DC) Warb. } & $\mathrm{G}$ & & & 0,1839 & 0,2665 & & 0,4064 & & & & 0,8568 \\
\hline & $\mathrm{N}$ & & & 1 & 1 & & 1 & & & & 3 \\
\hline \multirow[t]{2}{*}{ Eperua glabiflora (Ducke) R.S. Cowan } & G & 0,0645 & & 0,2843 & 0,1987 & & & & & & 0,5474 \\
\hline & $\mathrm{N}$ & 1 & & 2 & 1 & & & & & & 4 \\
\hline \multirow[t]{2}{*}{ Dinizia excelsa Ducke } & $\mathrm{G}$ & & & & & & & & & 1,4037 & 1,4037 \\
\hline & $\mathrm{N}$ & & & & & & & & & 1 & 1 \\
\hline \multirow[t]{2}{*}{ Pouroma myrmecophila Ducke } & $\mathrm{G}$ & 0,0907 & 0,2717 & & & & & & & & 0,3624 \\
\hline & N & 2 & 3 & & & & & & & & 5 \\
\hline Pouteria guianensis Aubl. & $\mathrm{G}$ & 0,0509 & 0,0812 & 0,3581 & & & & & & & 0,4902 \\
\hline & N & 1 & 1 & 2 & & & & & & & 4 \\
\hline $\begin{array}{l}\text { Licania octandra (Hoffmanns ex R. \& S.) } \\
\text { Kuntze }\end{array}$ & G & & 0,1979 & 0,2567 & & & & & & & 0,4546 \\
\hline & $\mathrm{N}$ & & 2 & 2 & & & & & & & 4 \\
\hline Piptadenia suevolens Miq. & G & & & & & & & & & 1,2732 & 1,2732 \\
\hline & $\mathrm{N}$ & & & & & & & & & 1,0000 & 1,0000 \\
\hline Total & $\mathrm{G}$ & 2,2681 & 2,9030 & 2,7465 & 2,1490 & 0,6217 & 0,4064 & 0,0000 & 0,0000 & 7,6850 & 18,7797 \\
\hline & N & 43 & 31 & 18 & 9 & 2 & 1 & 0 & 0 & 6 & 110 \\
\hline
\end{tabular}


vegetais, dentre outras informações, são fundamentais para o planejamento e o sucesso das açôes de seleção de árvores matrizes em comunidades vegetais na Amazônia.

\section{BIBLIOGRAFIA CITADA}

Amaral, I.L. 1996. Diversidade florística em floresta de terra firme, na regiāo do rio Urucu-AM. Dissertação de Mestrado, Instituto Nacional de Pesquisas da Amazônia/Universidade Federal do Amazonas, Manaus, Amazonas. 121pp.

Brazão, J.E.M.; Santos, M.M; Silva, Z.L. 1993. Vegetação e Recursos Florísticos.In: Caldeiron, S.S. (Ed.). Recursos Naturais e Meio Ambiente: Uma visão do Brasil. Vol 1. Fundação Instiuto Brasileiro de Geografia e Estatística-IBGE . Rio de Janeiro, RJ. p. $59-68$

Cronquist, A. 1981. An integrated system of classification flowering plants. Columbia University Press, N. York, USA. 1262pp.

Espírito-Santo, F.D.B.; Shimabukuru, Y.E.; Aragão, L.E.O.C; Machado, E.L.M. 2005. Análise da composição florística e fitossociologia da Floresta Nacional do Tapajós com apoio geográfico de imagens de satélites. Acta Amazonica, 35(2):155173.

Ferreira, L.V.; Prance, G.T. 1998. Species richness and floristic composition in four hectares in the Jaú National Park in upland forests in Central Amazonia. Biodiversity and Conservation, 7: 1349-1364.

Houghton, R. A.; Skole, D.L.; Nobre, C.A.; Hackler, J.L.; Lawrence, K.T.; Chomentowski, W.H. 2000. Annual fluxes of carbon from deforestation and regrowth in the Brazilian Amazon. Nature, 403:301-304.

Instituto Brasileiro de Geografia e Estatística-IBGE. 1999. Mapa digital temático de vegetação-Banco de dados SIPAM.

Knight, D.H. 1975. A phytosociological analysis of species-rich tropical forest on Barro Colorado Island, Panama. Ecological Monographs, 45:259-28.

Krebs, C.J. 1989. Ecological methodology. Harper \& Row, New York, USA. 654pp.

Lima Filho, D.A.; Matos, F.D.A.; Amaral, I.L.; Revilla, J.; Coêlho, L.S.; Ramos, J.F.; Santos, J.L. 2001. Inventário florístico de floresta ombrófila densa de terra firme, na região do Rio UrucuAmazonas, Brasil. Acta Amazonica, 31:565-579.

Magurran, A.E. 1988. Ecological diversity and its measurement. Princeton University Press, New Jersey, USA, 192pp.

Matos, F.D.A.; Amaral, I.L. 1999. Análise ecológica de um hectare em floresta ombrófila densa de terra-firme, estrada da várzea, Amazonas, Brasil. Acta Amazonica, 29:365-379.

Mobot, Missouri Botanical Garden. W ${ }^{3}$ Tropicos. Disponível em: http:// mobot.mobot.org /W3T/search/vast.html. Acesso: 2005.

Mori, A.S.; Boom, B. 1983. Ecological importance of Myrtaceae in an eastern Brazilian wet forest. Biotropica, 15:68-70.

Müller-Dombois, D.; Ellemberg, H. 1974. Aims and methods for vegetation ecology. John Wiley \& Sons, New York, USA. $547 \mathrm{pp}$.
Nelson, B.N.; Oliveira, A. 2001. Estado do conhecimento florístico da Amazônia. In: Capobianco, J.P.R.; Veríssimo, A.; Moreira, A.; Sanwyer, D.; Santos, I.; Pinto, L.P. (Eds.) Biodiversidade na Amazônia Brasileira: avaliação e açôes prioritárias para a conservação, uso sustentével e repartição de beneficios. (documentos temáticos). São Paulo. P. 132-182.

Nobre, C. A.; Sellers, P.; Shukla, J. 1991. Amazonian deforestation and regional climate change. Journal of Climate, 4: 957-988.

Oliveira, A.A.; Mori, S.A. 1999. A Central Amazonian terra firme forest. High tree species richness on poor soils. Biodiversity and Conservation, 8:1219-1244.

Oliveira, A.N.; Amaral, I.L. 2003. Florística e fitossociologia de uma floresta de vertente na Amazonia central, Amazonas, Brasil. Acta Amazonica, 34(1):9-35.

Oliveira, A.N.; Amaral, I.L. 2005. Aspectos florísticos, fitossociológicos e ecológicos de um sub-bosque de terra firme na Amazônia Central, Amazonas, Brasil. Acta Amazonica,35: 1-16.

Pinto, A.C.M; Higuchi, N.; Iida, S.; Santos, J. Dos; Ribeiro, R.J.; Rocha, R.M.; Silva, R.P da. 2003. Padrão de distribuição espacial de espécies florestais que ocorrem na região de Manaus-Am. Cap. 1. In: Higuchi, N.; Santos, J.; Sampaio, P.T.B.; Marenco, R.A.; Ferraz, J. Sales, P.C. de; Saito, M. Matsumoto, S. (Eds.) Projeto Jacaranda - Fase II: Pesquisas florestais na Amazônia Central. Instituto Nacional de Pesquisas da Amazônia - INPA. Manaus, p. 1-20.

Pitman, N.C.A.; Terborg, J.W.; Silvan, S.R.; Nunes, P.V.; Neil, D.A.; Ceron, C.E. 2001; Palácios, W.A.; Aulestia, M. Dominance and distribution of tree species in upper Amazonia terra firme. Ecology 82(8):2101-2117.

Prance, G.T.; Rodrigues, W.A.; Silva, M.F. 1976. Inventário florestal de um hectare de mata de terra firme, $\mathrm{km} 30$ da estrada ManausItacoatiara. Acta Amazonica, 6(1):9-35.

RADAM, 1978. Programa de Integração Nacional. Levantamentos de Recursos Naturais. v.18 (Manaus) - Radam (projeto) DNPM, Ministério das Minas e Energia. Brasil. 626pp.

Regis, W.D.E. 1993. Unidades de Relêvo. In: Caldeiron, S.S. (Eds). Recursos Naturais e Meio Ambiente: Uma visão do Brasil. Vol 1. Fundação Instiuto Brasileiro de Geografia e Estatística-IBGE. RiodeJaneiro, RJ. p.39-45.

Ribeiro, J.E.L.S, Hopkins, M.J.G.; Vicentini, A.; Sothers, C.A.; Costa, M.A.S.; Brito, J.M.; Souza, M.A.D.; Martins, L.H.P.; Lohmann, L.G.; Assunção, P.A.C.L.; Pereira, E.C.; Silva, C.F.; Mesquita, M.R.; Procópio, L.C. 1999. Flora da Reserva Ducke. Guia de Identificação das Plantas Vasculares de uma Floresta de Terra-firme na Amazônia Central, Instituto Nacional de Pesquisas da Amazônia, Manaus, 793pp.

Recebido em 10/08/2005

Aceito em 14/03/2008 